\title{
Energy and Physical Capital: A Case of Non-classical Dynamics
}

\author{
Burcu Afyonoğlu Fazlığlu ${ }^{1}$. \\ Agustín Pérez-Barahona ${ }^{2}$. Çağrı Sağlam ${ }^{3}$
}

Accepted: 28 February 2018 / Published online: 26 March 2018

(C) Springer Science+Business Media B.V., part of Springer Nature 2018

\begin{abstract}
We study the importance of considering different energy requirements for physical capital and final good production in an overlapping generations (OLG) resource economy. In contrast to the standard OLG framework, but consistently with the empirical evidence, we assume that the accumulation of physical capital requires more energy than the production of consumption goods. Focusing on exhaustible energy resources, we find that OLG equilibria can exhibit "non-classical dynamics": the economy generates complex dynamics where, differing from the response predicted by the standard approach, resource prices may not increase monotonically. This result illustrates that the technological assumptions behind the energy inputs should be taken with caution, in particular on dynamic analyses involving exhaustible energy resources.
\end{abstract}

Keywords Exhaustible energy resources $\cdot$ Economic dynamics $\cdot$ Overlapping generations

JEL Classification Q31 - Q43 · O44

\section{Introduction}

The accumulation of physical capital has been frequently considered as a crucial ingredient to cope with the limitation to economic growth due to the usage of exhaustible energy resources (this literature dates back to the seminal papers of Dasgupta and Heal 1974, 1979; Solow 1974; Stiglitz 1974). However, the majority of this literature, assuming the same production technology for both consumption and physical capital goods, is inconsistent with

\footnotetext{
Agustín Pérez-Barahona

agustinperezbarahona@gmail.com

1 TOBB-ETU University, Ankara, Turkey

2 THEMA, Université de Cergy-Pontoise and Ecole Polytechnique, 33 Boulevard du Port, 95011

Cergy-Pontoise Cedex, France

3 Bilkent University, Ankara, Turkey
} 
the empirical evidence that identifies significant heterogeneity in the energy requirements of these two sectors. In this paper we investigate the implications of assuming different energy technologies for physical capital and final good production. More specifically, our study illustrates how the standard results regarding the dynamics of exhaustible resources, and their corresponding prices, are modified. We point out in this respect the intergenerational mechanisms behind the extraction of natural resources.

Analyzing the technological energy characteristics of the economy, data show significant heterogeneity among sectors. In particular, by means of considering energy intensity ratios, several recent studies (e.g., Ecorys 2009; Upadhyaya 2010; UNIDO 2011) find that the sectors involved in the formation of physical capital, such as manufacturing sectors in the construction of infrastructures and buildings, are highly energy-intensive. In contrast to this, manufacturing sectors that are related to consumption goods are considered as moderate or low energy-intensive. Moreover, from a panel analysis of 39 countries (OECD, Asia and Latin America) between 1971 and 1996, Miketa (2001) concludes that the physical capital formation has generally led to an increase of the energy intensity of the economy. Papers such as Bullard and Herendeen (1975) and Costanza (1980) have already pointed out the importance of explicitly considering a distinct energy technology to produce physical capital goods. With regard to this issue, Costanza (1980) also suggests that energy resources could seriously limit economic growth through the process of physical capital accumulation.

To the best of our knowledge, assuming different energy technologies in the production of physical capital and consumption goods has been only studied in Pérez-Baharona (2011). Considering a general equilibrium model with infinite-horizon representative household, this paper allows for a richer extraction dynamics of the exhaustible energy resource: if the physical capital technology requires relatively more energy than the production of consumption goods, resource extraction decreases in time but not monotonically. However, due to the complexity of his analysis (applying a method of Special functions), the underlying economic mechanisms remain ambiguous. In our paper we consider the overlapping generations (OLG) framework, which is a tractable alternative to the infinite-horizon representative agent models (Acemoglu 2009). Moreover, numerous papers state that the OLG approach offers a clearer explanatory capacity for natural resource problems, allowing us to understand the contribution of the intergenerational dimension. ${ }^{1}$ Pérez-Baharona (2011) additionally assumes that the production technology of consumption goods is linear (i.e., an AK technology). From this dimension, our paper also extends his framework to the case of non-linear production function.

In the literature on natural resources and OLG models, the behaviour of the economy over time typically exhibits (asymptotic) convergence to a single steady-state (or a balanced growth path). Under linear (or without) regeneration of resources and with exogenous (or no) technological progress, this long-run equilibrium is saddle-path stable and, consequently, the equilibrium trajectory is unique. The standard results about economic growth and the dynamics of exhaustible energy resources in OLG economies are mainly built upon the frameworks of Mourmouras (1991) and Olson and Knapp (1997). They show that, without technical progress and under standard assumptions about preferences and technology, the path of resources extraction exhibits a monotonic behaviour, decreasing over time towards

1 This literature frequently refers to the following three main reasons to prefer OLG to infinitely-lived agent models. First, resources are a store of value between generations (see Koskela et al. 2002; Valente 2008; BednarFriedl and Farmer 2013). Second, intergenerational aspects should be taken into account when analyzing environmental issues and/or natural resource economies (Solow 1974; Padilla 2002; Agnani et al. 2005). Finally, there exists empirical evidence showing that agents are not perfect altruistically linked (among others, Altonji et al. 1992; Balestra 2007). 
the steady-state corresponding to natural resources exhaustibility. Olson and Knapp (1997) notice though that, under non-standard assumptions (finite horizon quadratic utility), the convergence to this steady-state does not need to be monotone over time. Indeed the extraction can transitory rise, with a subsequent short-run decrease of the resources prices. Moreover, cycle extraction is shown to be numerically possible under infinite horizon, CES preferences and for a particular set of parameters. Our paper contributes to the literature by showing that the economy generates complex dynamics and, consequently, resources extraction and energy prices can evolve non-monotonically over time. In contrast to those approaches, our conclusion does not rely on non-standard preferences or any particular parameterizations of the exogenous functions involved in the model. Furthermore, this dynamic result is fully analytical.

Our set-up mainly follows the OLG framework introduced by Agnani et al. (2005), aiming at extending the model of Pérez-Baharona (2011). Focusing on exhaustible resources, we describe the global dynamic properties of the economy with respect to the share of energy inputs in the production of physical capital. As usual, the stock of exhaustible resources monotonically decreases over time. However, in contrast to Mourmouras (1991), the resource extraction does not necessary decrease following a monotonic trajectory if the share of energy in the production of physical capital is low enough. As a consequence, differing from the "classical behavior" predicted by the standard approach built on Hotelling's (1931) seminal work, resource prices can decrease in the short-run.

Importantly, the empirical literature on non-renewable resources already identifies the discrepancy between Hotelling's prediction and the empirical evidence about resource prices, including energy resources. ${ }^{2}$ For instance, mineral commodity prices show relative declines and fluctuation around time trends rather than persistent increases (see figures 1-11 in Krautkramer 1998). Considering a large data set of U.S. mineral prices, Barnett and Morse (1963) were the first to systematically identify this pattern. Slade (1982) further reveals that a U-shaped trajectory is more appropriate in fitting the observed prices of many non-renewable resources over the period 1870-1978 (see Ferraro and Peretto 2014, for a recent study). From a theoretical perspective, known examples of channels that can generate non-monotonic trends are backstop technologies (Heal 1976), informational asymmetries (Pindyck 1980), technical change (Slade 1982), the role played by environmental constraints and natural resource abundance (Ahrens and Sharma 1997), or the transitions between renewable and non-renewable energy resources (Tahvonen and Salo 2001). Our paper identifies in this regard new ingredients to understand the "non-classical dynamics" of natural resource prices, namely the technological energy characteristics of the formation of physical capital and its interaction with the intergenerational transmission of natural resources.

The paper proceeds as follows. The general model is introduced in Sect. 2, together with the definition of competitive equilibrium. Section 3 presents the corresponding equilibrium dynamics and examines the stability properties of the economy, paying particular attention to the case of exhaustible energy resources. Conclusions and possible extensions of the paper are discussed in Sect. 4.

\section{The Model}

We consider a perfect foresight overlapping generations economy, without population growth, in discrete time with infinite horizon. In contrast to the standard OLG approach, our model assumes that physical capital accumulation and production of consumption goods have dif-

2 See Krautkramer (1998) for a discussion of this literature. 
ferent energy requirements. More precisely, we follow the empirical evidence supporting that the physical capital sector is more energy-intensive than the consumption good sector. As Pérez-Baharona (2011) and Le and Le Van (2016, 2017), we will focus on the example of an economy where only the production of physical capital requires energy. Our framework is a highly stylized model of the economy, which aims at illustrating the main mechanisms behind sectoral differences on energy requirement. Neglecting for the endogenous allocation of energy between sectors is not realistic. Nevertheless, the greater analytical tractability of this rather extreme case will allows us to identify the key ingredients of the problem.

We assume an economy with three sectors: final good production, physical capital, and extraction sectors. The final good, which can be either directly consumed or invested, is obtained by combining labor and the current stock of physical capital. As usual, each household provides one unit of labor. The physical capital sector produces the capital of the economy, where investment and energy are the corresponding technological inputs. Finally, the extraction sector directly supplies the energy from the extraction of natural resources. We consider that all agents have rational expectations and each generation consists of $N_{t}>0$ identical households. Moreover, they are price-takers and all the markets are considered to be competitive.

Since we will assume that the households own the natural resources, let us first specify the extraction sector and the consumers' problem. We then describe the firms and the competitive equilibrium of the economy, setting the optimal behavior of final good and physical capital sectors.

\subsection{Extraction Sector}

Following Agnani et al. (2005), natural resources are considered to be an asset (i.e., store of value) and a technological input. In our model, the economy uses both energy and investment inputs in order to produce the physical capital. As in their paper, we assume a grandfathering regime, so that the initial old generation possesses the whole stock of natural resources. Moreover, at the beginning of each period $t$ the current old agents own the resource stock $E_{t}$. This is defined as $E_{t} \equiv e_{t} N_{t}$, where $e_{t}$ is the amount of resources per-worker. $N_{t}$ represents the number of workers (households) of generation $t$ in the economy, which can grow at an exogenous rate $n \geq 0$, i.e., $N_{t+1}=(1+n) N_{t}$.

For simplicity, as in Mourmouras (1991) or Olson and Knapp (1997), we consider that the old agents extract themselves the natural resources at zero cost (we specify the consumers' problem in Sect. 2.2). ${ }^{3}$ They can directly sell those resources, as energy input, to the physical capital sector. The extraction flow is denoted by $X_{t}$, and the remaining part of the natural resource is sold to the young agents as a resource asset $A_{t}$ (i.e., $A_{t}=E_{t}-X_{t}$ ). Following the general formulation of Mourmouras (1991), the resource stock regenerates at a linear rate $\Pi \geqslant 1$ from period $t$ to $t+1$. The law of motion of the stock of energy resources, in per-worker terms, can be formalized as follows:

$$
e_{t+1}=\Pi\left(e_{t}-x_{t}\right)=\Pi a_{t},
$$

where $x_{t} \equiv X_{t} / N_{t}$ and $a_{t} \equiv A_{t} / N_{t}$. Notice that, for the shake of exposition, we also consider that population is constant in our economy and, then, $n=0$. As pointed out in the introduction, we will focus on the dynamic properties of non-renewable resources. We should notice however that, for the generality of our approach, we present a model that allows for both types of resources, namely renewable $(\Pi>1)$ or exhaustible $(\Pi=1)$.

\footnotetext{
3 Our model does not include a more detailed specification of this sector because issues such as the technical progress in extraction or the effect of market power are beyond the scope of the paper.
} 


\subsection{Households}

Each consumer receives an income $w_{t}$, which is equal to the real wage from supplying her single unit of labor to the final good firm when young. She then allocates this income among the current consumption $c_{t}$, the savings $s_{t}$ related to investment, and the purchase of $a_{t}$ ownership rights of natural resources. In her last period of life (when she is old at period $t+1$ ), the agent does not work and just consumes $d_{t+1}$ out of her entire income, not leaving any bequests. Her income in $t+1$ is generated from the return on savings made when she was young $R_{t+1} s_{t}$, where $R_{t+1} \equiv 1+r_{t+1}$ and $r_{t+1}$ is the interest rate. Moreover, she also gets income from extracting natural resources and offering them as energy input to the physical capital sector $Q_{t+1} x_{t+1}$, and from selling the rest to the young households $P_{t+1} a_{t+1}$, where $Q_{t+1}$ and $P_{t+1}$ are the corresponding prices. Accordingly, the budget constraints that a consumer of generation $t$ faces are:

$$
\begin{aligned}
c_{t}+s_{t}+P_{t} a_{t} & =w_{t}, \\
d_{t+1} & =R_{t+1} s_{t}+Q_{t+1} x_{t+1}+P_{t+1} a_{t+1} .
\end{aligned}
$$

Generations derive utility from consumption, where their two-period intertemporal utility function depends on the level of consumption when young $c_{t}$ and when old $d_{t+1}$. We assume an additively separable life-cycle utility function $U\left(c_{t}, d_{t+1}\right)=u\left(c_{t}\right)+\beta u\left(d_{t+1}\right)$, where $\beta \in(0,1)$ is the subjective discount factor. In particular, we adopt a logarithmic instantaneous utility function $u(\cdot)$ since we are mainly concerned with the existence of the competitive equilibrium and its qualitative properties.

Taking the prices of the energy resource and wages as given, the agent born at time $t$ maximizes her utility by choosing young and old period consumption, and the ownership rights of natural resources. The corresponding optimization problem of the consumer is formalized as follows:

$$
\max _{\left\{c_{t}, d_{t+1}, s_{t}, e_{t+1}\right\}} \ln c_{t}+\beta \ln d_{t+1}
$$

subject to

$$
\begin{aligned}
c_{t}+s_{t}+P_{t} a_{t} & =w_{t}, \\
d_{t+1} & =R_{t+1} s_{t}+Q_{t+1} x_{t+1}+P_{t+1} a_{t+1}, \\
e_{t+1} & =\Pi\left(e_{t}-x_{t}\right) \\
c_{t} & \geq 0, d_{t+1} \geq 0, e_{t+1} \geq 0, E_{0}>0 \text { given. }
\end{aligned}
$$

We then obtain the following first-order conditions (FOC):

$$
\begin{aligned}
\frac{d_{t+1}}{c_{t}} & =\beta R_{t+1}, \\
\frac{P_{t+1}}{P_{t}} & =\frac{R_{t+1}}{\Pi}, \\
P_{t+1} & =Q_{t+1} .
\end{aligned}
$$

Equation (4) equalizes the discounted marginal utilities, where the marginal rate of substitution between current and future consumption is equal to their relative prices. Equation (5) is the non-arbitrage condition between the different types of savings (one is related to investment and the other to ownership rights). Finally Eq. (6) is an additional non-arbitrage condition, which implies that in the equilibrium the prices of the asset and of the extracted energy are the same. This last result is indeed consistent with Olson and Knapp (1997) and 
Valente (2008). Moreover, notice that Eqs. (5) and (6) are equivalent to the Hotelling rule presented in Mourmouras (1991).

\subsection{Firms}

\subsubsection{Final Good Sector}

The firms in this sector produce the final good be means of a Cobb-Douglas technology. Equation (7) below specifies the production function at any date $t$, where $Z_{t}$ (Eq. 8) represents the (exogenous) total factor productivity. With constant returns to scale, the number of firms does not matter and the production is independent of the number of firms that use the same technology. We are then concerned with the problem of a representative firm. Under this perfectly competitive environment, taking the prices of inputs $\left(P_{t}^{K}\right.$ and $\left.w_{t}\right)$ and the initial levels of technology and capital stock as given, the representative firm maximizes at each period $t$ the profit by choosing the amount of labor and physical capital inputs:

$$
\max _{\left\{K_{t}, N_{t}\right\}} Y_{t}-P_{t}^{K} K_{t}-w_{t} N_{t}
$$

subject to

$$
\begin{array}{rlrl}
Y_{t} & =Z_{t} K_{t}^{\alpha} N_{t}^{1-\alpha} & & 0<\alpha<1, \\
Z_{t+1} & =(1+z) Z_{t} & z \geq 0 .
\end{array}
$$

At an interior solution of the firm's optimization problem the following FOCs are satisfied. Input prices equal the corresponding marginal benefits, where all variables are expressed in per-worker terms $\left(k_{t} \equiv K_{t} / N_{t}\right.$ and $\left.y_{t} \equiv Y_{t} / N_{t}\right)$ :

$$
\begin{aligned}
\alpha y_{t} & =P_{t}^{K} k_{t}, \\
(1-\alpha) y_{t} & =w_{t} .
\end{aligned}
$$

Equation (11) below summarizes the market clearing condition of the economy. As pointed out before, the final good in $t$ is consumed by young and old (generation $t-1$ ) agents, $C_{t}$ and $D_{t}$ respectively, or invested for the production of future capital stock as savings $S_{t}$ :

$$
Y_{t}=C_{t}+D_{t}+S_{t}
$$

\subsubsection{Physical Capital Sector}

This sector provides the physical capital to the economy. In our model physical capital is considered to be an intermediate good. The standard OLG approach assumes that the capital stock at time $t+1$ is entirely determined by the savings made at time $t$, which is equal to the investment. Notice that this literature frequently focuses on the case of full depreciation since every period $t$ represents a whole life stage of an individual, i.e., typically around 30 years (for instance, Acemoglu 2009). Thus $K_{t+1}=I_{t}$. If energy were a production factor of the economy, this expression together with (11) implies that the production of each unit of physical capital or consumption goods would have the same energy requirements. However, the empirical evidence shows that the physical capital production is relatively more energy-intensive than consumption. As observed before, we illustrate this idea by means of considering that only physical capital requires energy to be produced. Then the formation of 
physical capital will be determined not only by the investment but also by the energy that this process requires. ${ }^{4}$

In contrast to previous OLG models, the physical capital at $t+1$ is produced by means of combining the extracted energy resources $X_{t+1}$ and the investment $I_{t}$. Assuming a CobbDouglas technology, 5

$$
\begin{aligned}
K_{t+1} & =B_{t+1}^{\theta} X_{t+1}^{\theta} I_{t}^{1-\theta} & & 0<\theta<1, \\
S_{t} & =I_{t}, & & \\
B_{t+1} & =(1+b) B_{t}, & & b \geq 0 .
\end{aligned}
$$

Savings equals investment in our model (Eq. 13). The investment made in $t$ is used to built the capital in the next period $t+1$. But this time investment is considered as an input, together with energy, to create new physical capital (Eq. 12). $\theta \in(0,1)$ represents the importance of natural resources for the physical capital formation. As in the standard OLG literature, we assume full depreciation of physical capital since each period $t$ represents a long time span. The scale parameter $B$ grows at a rate $b$, representing the exogenous technical progress in the production of capital goods. We consider that the capital used in $t+1$ is produced with the available technology $B_{t+1}$ at the beginning of the period (Eq. 14). Consistently with this assumption, the production of $K_{t+1}$ requires the extracted energy of resources $X_{t+1}$. Notice finally that, in contrast to (8), the technical progress in this sector is energy-saving and specific to the formation of physical capital.

The representative firm of this sector maximizes profits at each period $t$, choosing the amount of energy resources that will be used in the formation of physical capital:

$$
\max _{\left\{X_{t}\right\}} P_{t}^{K} K_{t}-Q_{t} X_{t}
$$

subject to

$$
K_{t}=B_{t}^{\theta} X_{t}^{\theta} I_{t-1}^{1-\theta},
$$

taking as given the prices of capital and energy, and the level of investment. At an interior solution of the firm's optimization problem the following FOC is satisfied:

$$
Q_{t}=\theta P_{t}^{K} B_{t}^{\theta} X_{t}^{\theta-1} I_{t-1}^{1-\theta} .
$$

Therefore, taking the technologies (7) and (12), and the price condition (9), Eq. (15) becomes:

$$
Q_{t}=\theta \alpha Y_{t} X_{t}^{-1} \text {. }
$$

As in Agnani et al. (2005), under full depreciation of physical capital, the return of investing in physical capital $R_{t} S_{t-1}$ at time $t$ should be equal to the profit of producing new capital $(1-\theta) P_{t}^{K} K_{t}$ to prevent arbitrage opportunities. ${ }^{6}$ We then obtain the following condition since $S_{t-1}=I_{t-1}$ :

\footnotetext{
${ }^{4}$ Ecorys (2009, p. 10) also points out that "most of the energy-intensive industries produce intermediate goods".

5 We take a Cobb-Douglas formulation in order to compare our results with the previous OLG literature, in particular with Mourmouras (1991) and Olson and Knapp (1997) that specifically consider this type of technology. Moreover, as Dasgupta and Heal (1979) observe, one can neglect the thermodynamic constraint of a minimum energy requirement (i.e., Leontief production function). This is possible if we interpret energy as an input service that already incorporates a basic level of energy (for an explicit modeling of this physical constraint see, for instance, Pérez-Barahona and Zou 2006).

${ }^{6}$ Choosing the optimal resource stock (15) and the technology (12), the maximum profit that the representative firm can obtain is given by
}

$$
\pi_{t}^{*}=P_{t}^{K} K_{t}-\theta P_{t}^{K} B_{t}^{\theta} X_{t}^{\theta-1} I_{t-1}^{1-\theta} X_{t}=(1-\theta) P_{t}^{K} K_{t} .
$$




$$
R_{t}=(1-\theta) P_{t}^{K} B_{t}^{\frac{\theta}{1-\theta}} X_{t}^{\frac{\theta}{1-\theta}} K_{t}^{\frac{\theta}{\theta-1}} .
$$

\subsection{The Competitive Equilibrium}

The dynamic competitive equilibrium for our OLG natural resource economy is defined as a sequence of prices $\left\{w_{t}, R_{t}, P_{t}^{K}, P_{t}, Q_{t}\right\}_{t=0}^{\infty}$ and feasible allocations $\left\{c_{t}, d_{t}, s_{t}, e_{t}, a_{t}, x_{t}, i_{t}, y_{t}, k_{t+1}\right\}_{t=0}^{\infty}$, given the positive initial values for $K_{0}, E_{0}, Z_{0}, B_{0}$, $N_{0}$, and the law of motion of exogenous technological progresses $\left(Z_{t}\right.$ and $\left.B_{t}\right)$ and population $\left(N_{t}\right)$, such that the consumers maximize their life-time utility, firms maximize their profits, and all markets clear at every period $t$. Considering the results presented above, this equilibrium is the solution of the system of Eqs. (1)-(17). ${ }^{7}$

\section{Equilibrium Dynamics}

The main focus of our paper is to study the dynamics of the economy around the steady-state equilibrium. We therefore assume that there is no technological progress, i.e., $b=z=0$ in (8) and (14). As in Mourmouras (1991), Farmer (2000) and Bednar-Friedl and Farmer (2013), we will show that the intertemporal equilibrium dynamics can be reduced to a twodimensional system. One of the equations represents the law of motion of the stock of natural resources $E_{t}$, while the other describes the evolution of the extraction flow $X_{t}$. We provide all computational details in "Appendix A".

Using the physical capital technology (12), the condition (13), the budget constraints and the FOCs (2)-(5) of the households' problem, and the FOC (10) of the firms' maximization problem, we obtain for $k_{t+1}$

$$
k_{t+1}^{\frac{1}{1-\theta}}=B_{t+1}^{\frac{\theta}{1-\theta}} x_{t+1}^{\frac{\theta}{1-\theta}}\left[\sigma(1-\alpha)+\theta \alpha\left(1-\frac{e_{t}}{x_{t}}\right)\right] y_{t},
$$

with $\sigma \equiv \beta /(1+\beta)$. In addition, taking (5), (6), (9), (15) and (17), the following difference equation for $k_{t+1}$ arises:

$$
\frac{\Pi k_{t+1}^{\frac{1}{1-\theta}} x_{t}}{y_{t} x_{t+1}}=\alpha(1-\theta) B_{t+1}^{\frac{\theta}{1-\theta}} x_{t+1}^{\frac{\theta}{1-\theta}}
$$

Substituting for $k_{t+1}$ in those expressions yields equation (20) below, which describes the dynamics of resource extraction. Since it also depends on the stock of resources $e_{t}$, we need to recall the law of motion of the natural resources (1) in order to set the following dynamical system:

\section{Footnote 6 continued}

Notice that the non-arbitrage condition $R_{t} S_{t-1}=(1-\theta) P_{t}^{K} K_{t}$ can be alternatively obtained as a FOC if we assume that the firm also decides about its demand of investment input $I_{t-1}^{d}$. We would then include the new term $-\left(1+r_{t}\right) I_{t-1}^{d}$ in the maximization problem above. $R_{t}$, which is defined as $1+r_{t}$, will adjust in order to ensure that $I_{t-1}=I_{t-1}^{d}$.

7 It is customary to assume in this literature (e.g., de la Croix and Michel 2004) that, at the first period $t=0$, each old household $N_{-1}$ is the owner of the same fraction $s_{-1}$ of installed capital stock $K_{0}: s_{-1}=K_{0} / N_{-1}$. Considering (12) and (13), one can obtain an equivalent expression for our model where energy is required to produce physical capital: $s_{-1}=K_{0}{ }^{1 / \theta} / X_{0} N_{-1}$. 


$$
\begin{aligned}
& x_{t+1}=\Pi\left(\frac{\frac{\beta}{(1+\beta)}(1-\alpha)+\alpha \theta}{\alpha(1-\theta)}\right) x_{t}-\frac{\Pi \theta}{(1-\theta)} e_{t}, \\
& e_{t+1}=\Pi\left(e_{t}-x_{t}\right) .
\end{aligned}
$$

The linear planar system (20)-(21) describes the dynamics of our economy, which can be rewritten in matrix form. The next proposition summarize this outcome:

Proposition 1 The dynamic properties of our economy are described by the system of difference equations

$$
\left[\begin{array}{l}
x_{t+1} \\
e_{t+1}
\end{array}\right]=\Psi\left[\begin{array}{l}
x_{t} \\
e_{t}
\end{array}\right],
$$

where we define the matrix $\Psi \equiv\left[\begin{array}{ll}\psi_{1} & \psi_{2} \\ \psi_{3} & \psi_{4}\end{array}\right]$ with

$$
\begin{aligned}
& \psi_{1} \equiv \frac{\Pi}{(1-\theta)} \frac{\beta}{(1+\beta)} \frac{(1-\alpha)}{\alpha}+\frac{\Pi \theta}{(1-\theta)}, \\
& \psi_{2} \equiv-\frac{\Pi \theta}{(1-\theta)} \\
& \psi_{3} \equiv-\Pi \\
& \psi_{4} \equiv \Pi .
\end{aligned}
$$

\subsection{Steady-State Equilibria}

Let us first identify the steady-state equilibria of our economy. In this respect, we provide the necessary and sufficient conditions for existence of such equilibrium:

Proposition 2 The steady-state equilibria $\left(x^{*}, e^{*}\right)$ of our economy are the solutions of the following system of equations:

$$
\begin{aligned}
& x^{*}=\psi_{1} x^{*}+\psi_{2} e^{*}, \\
& e^{*}=\psi_{3} x^{*}+\psi_{4} e^{*} .
\end{aligned}
$$

We can then conclude that there is a continuum of steady-states iff $\left\{\psi_{1} \neq 1, \psi_{4} \neq 1\right.$ and $\left.\left(1-\psi_{4}\right)=\frac{\psi_{2} \psi_{3}}{\left(1-\psi_{1}\right)}\right\}$, where $x^{*}=\frac{\psi_{2}}{1-\psi_{1}} e^{*}$. Otherwise, there is a unique steady-state $\left(x^{*}, e^{*}\right)=(0,0)$.

Proof See "Appendix B".

As it is clear from Proposition 2, the economy has either a unique steady-state $\left(x^{*}, e^{*}\right)=$ $(0,0)$ or a continuum of them. As $\psi_{4}=1$ for non-renewable resources (i.e., $\left.\Pi=1\right)$. Then, the only possible steady-state for this type of resources would $\left(x^{*}, e^{*}\right)=(0,0)$. This is the usual result with non-renewable resources, implying their long-run exhaustibility. In the next section, we will study how the economy converges (if that is the case) to the steady-state.

\subsection{Stability and Exhaustible Resources}

As observed in the introduction, our general objective is to highlight the role played by the energy intensity assumptions of the different sectors of the economy. We will demonstrate in this section that those assumptions have important dynamic implications and, therefore, 
they should be taken with caution. In particular, we will show that the different energy requirement of the physical capital and consumption goods sectors turns out to be relevant to understand economic dynamic phenomena such as the non-classical behavior of exhaustible energy resources. We will use in this regard the general framework presented in Sect. 2, paying attention to the case of non-renewable resources.

From now on, we will concentrate on non-renewable resources and the dynamics of the economy around the corresponding unique steady-state $\left(x^{*}, e^{*}\right)=(0,0) .{ }^{8}$ We analyze below the stability properties of the system and the subsequent possibility of non-monotone convergence. The model will allow us to identify the fundamental role played by the share of energy in the capital accumulation, together with its interaction with the discount rate and the share of physical capital in the final good production. Notice as well that our conclusions are of global dynamics since the system is linear and, therefore, we do not need to apply any approximation around the steady-state.

\subsubsection{Dynamics}

We denote by $\lambda_{1}$ and $\lambda_{2}$ the eigenvalues of matrix $\Psi$ in Proposition 1. Let us define the combination of parameters $\tilde{\beta} \equiv \frac{\beta}{(1+\beta)} \frac{(1-\alpha)}{\alpha}>0$. The following proposition entirely describes the dynamics of our economy by means of studying the eigenvalues $\lambda_{1}$ and $\lambda_{2}$. In particular, we identify cases where complex dynamics arise, allowing our model to reproduce the nonclassical response described in the introduction. These conditions prove to be related to the assumption of considering that the physical capital sector is more energy intensive than the final good production.

Proposition 3 For exhaustible resources and different parameter combinations, the stability of the zero steady-state changes such that:

(i) For $0<\theta<\frac{1}{4}$ and $\tilde{\beta}+\frac{1}{\tilde{\beta}}<2(1-2 \theta)$ the dynamics of the economy are complex:

(a) If $\theta<1-\tilde{\beta}$ both eigenvalues (in absolute value) are smaller than one, so the steady-state is stable and indeterminacy occurs.

(b) If $\theta>1-\tilde{\beta}$ both eigenvalues (in absolute value) are greater than one, so the steady-state is unstable.

(ii) Otherwise, the dynamics are non-complex. Moreover in this case the eigenvalues are on the different side of one $\left(\lambda_{1}>1\right.$ and $\left.0<\lambda_{2}<1\right)$ and, therefore, the steady-state is saddle-path stable.

Proof See "Appendix C".

Proposition 3 shows that the share of energy resource input in the formation of physical capital (represented in the model by the parameter $\theta$ ) is key to understand the dynamics of the economy. When the share of energy in the accumulation of physical capital is high enough $\left(\frac{1}{4} \leq \theta<1\right)$ we get non-complex saddle-path dynamics. This implies, for non-renewable resources, the usual monotonic asymptotic convergence to exhaustibility. However, for a lower share $\left(0<\theta<\frac{1}{4}\right)$ the economy can reproduce situations of complex dynamics and, in particular, of non-monotone convergence. This result is presented in the statement (i.a) of the proposition. Since energy is assumed to be non-renewable in this economy, the stock

8 We provide a discussion about the case of renewable resources in the concluding remarks. 
of resources $e_{t}$ cannot increase with time. However, differing from the situation of noncomplex dynamics, the extraction (flow) of resources $x_{t}$ does not necessary decrease all time during the (asymptotic) convergence to the zero steady-state. This result is consistent with the non-monotonic convergence result of Pérez-Baharona (2011), where the production of consumption goods is assumed to be linear (AK technology). From this perspective, our paper extends his conclusion to the case of non-linear technology and OLG framework.

We should also observe that the dynamics described above can somehow give support to the Environmental Kuznet Curve (EKC). ${ }^{9}$ If one associates increasing extraction with environmental degradation, equation (18) and the complex dynamics of extraction show the possibility of a non-monotonic correlation between extraction and income per capita. If extraction $\left(x_{t+1}\right)$ increases, income per capital $\left(y_{t+1}=Z k_{t+1}^{\alpha}\right)$ raises too. It is however possible that, after some periods, income per capital increases even if extraction reduces: this can happen if the effect of a lower extraction $x_{t+1}$ is compensated by a greater $y_{t}$ and, thus, a higher investment and creation of physical capital.

Let us finally relate the evolution of the energy prices with the dynamics of the extraction. This can be done by replacing $Y_{t}$ in (16) by the technology (7), and then $K_{t}$ by (12). Hence, we can state the following:

Proposition 4 Provided that $\alpha, \beta \in(0,1)$, there is a negative relationship between extraction and energy prices $Q_{t}$.

Considering the extraction dynamics, Proposition 4 allows us to conclude that energy prices can transitory decrease. This evolution of extraction and resource prices corresponds to the non-classical response of exhaustible energy resources, which was empirically verified by previous studies. From a theoretical point of view, the possibility of non-monotonic convergence is an important property of our framework since it provides an alternative explanation to this empirical fact. It underlines the influence of the energy intensity assumptions on the dynamic predictions of the model. Moreover, we should notice that this non-classical dynamics is likely to happen for small values of $\theta$ (lower than $1 / 4$ in our model), which corresponds to economies with a relatively low share of energy inputs in the production of physical capital. This situation is in line with the improvement of energy efficiency that was empirically identified in the industrial sector of many developed countries (among others, Miketa 2001; Ecorys 2009; UNIDO 2011).

\subsubsection{Economic Mechanisms}

Proposition 3 provides necessary and sufficient conditions for complex/non-complex dynamics. However, regarding to specific non-monotonic dynamics such as the EKC, the U-shaped evolution of energy prices (Slade 1982), or cycles (e.g., Kim and Lounging 1992; Olson and Knapp 1997; Lutz 2008), one should identify further particular conditions. Nevertheless, even if from this perspective our conditions are only necessary, they already allow us to identify key economic mechanisms to understand those types of non-classical dynamics.

As pointed out before, $\theta$ is an important parameter for the dynamics of the economy. It is closely related to the importance that each generation gives to the energy resources as an input for the formation of physical capital. This will affect in turn the value of the ownership rights of natural resources $\left(a_{t}\right)$, which are sold from one generation to the next. In economies where $\theta$ is small $(0<\theta<1 / 4)$ energy resources are relatively unimportant with respect

9 The EKC hypothesis postulates that environmental quality deteriorates at early stages of development and subsequently improves afterwards (for instance, Kijima et al. 2010). 
to the other inputs in the physical capital technology. Therefore, the future generations give little value to the ownership rights of resources. The market would then not have incentives to leave many resources to the next generation, increasing thus the extraction made by the old generation. ${ }^{10}$ This will continue until energy becomes scarce enough. At this moment the value of the ownership rights raises, creating new incentives to reduce extraction and, consequently, increasing the amount of resources left to the next generation.

Notice too that the occurrence of non-classical dynamics depends as well, through the coefficient $\tilde{\beta}$ defined before, on the share of physical capital in the technology of final good $(\alpha)$ and on the discount rate $(\beta)$. Statement (i) in Proposition 3 identifies boundaries for $\tilde{\beta}$. On the one hand, the condition in (a) can be rewritten as $\tilde{\beta}<1-\theta$, providing an upper limit. ${ }^{11}$ This implies that $\tilde{\beta}<2(1-2 \theta)$ since $(1-\theta)<2(1-2 \theta)$ for $0<\theta<1 / 4$. On the other hand, the second condition $\tilde{\beta}+1 / \tilde{\beta}<2(1-2 \theta)$ states then that $\tilde{\beta}$ cannot be too small either because of the term $1 / \tilde{\beta}$.

In order to study the effect of these boundaries and, then, the role played by $\alpha$ and $\beta$, let us consider a reference economy where the values for these parameters are such that the conditions of statement (i.a) hold. For a higher discount rate the parameter $\tilde{\beta}$ will increase and, therefore, the upper limit may not be respected anymore. However, this effect can be counterbalanced if the economy has a higher share of physical capital in the technology of final goods. ${ }^{12}$ The economic interpretation is the following. A higher discount rate implies greater concern of consumers for their old age and, consequently, for the amount of resources they are going to extract or leave (sell) to the next generation. Since energy is not very important in this economy $(0<\theta<1 / 4)$, the next generation will not value much the ownership rights. Therefore, as in the mechanism identified before, the current generation may increase the resource extraction for some periods. Nevertheless, a larger share of capital in final good technology would increase the value that future generations attach to the energy resources and the corresponding ownership rights, ensuring then that the extraction eventually decreases.

Let us finally observe that the economic mechanisms identified here are in agreement with papers such as Brookes (2000), Greening et al. (2000) and Sen (2016). These papers identify the possibility of having an increasing energy extraction (supply) that is compatible with situations where the technological energy requirements have been reduced (a small $\theta$ in the context of our paper). Brookes (2000) and Greening et al. (2000) provide an example within the context of the implementation of energy efficiency policies, while Sen (2016) takes the international trade perspective. With respect to this literature, our paper shows a new channel that is based on the interaction between two important elements: the energy intensity differences among sectors and the intergenerational transmission of energy resources.

\section{Concluding Remarks}

Using an OLG natural resources framework, we examine the dynamic implications of assuming different energy technologies for physical capital and final good production. We take advantage of the analytical tractability of our highly stylized model, where only the formation of physical capital requires energy. We find that the introduction of energy-intensity

\footnotetext{
10 Since consumers are non-altruistic and live for two periods, the existence of ownership rights prevent the old generation from extracting all resources.

11 Under condition (b) the zero steady-state is unstable. However, due to the non-negativity condition of the stock of resources and extraction, the economy exhausts all natural resources. In contrast to (a), this would happen in finite time instead of asymptotically.

12 From the definition of $\tilde{\beta}$, it is easy to see that $\partial \tilde{\beta} / \partial \beta>0$ and $\partial \tilde{\beta} / \partial \alpha<0$.
} 
differentiation among sectors has important implications for the standard results in the area. Richer dynamics other than saddle arise. Focusing on exhaustible resources, our economy can reproduce non-classical dynamics. Energy extraction and prices do not necessary follow a monotonic trend over time, which is an outcome consistent with previous empirical findings. The model allows us to identify new ingredients that can improve our understanding of this puzzling issue, namely the interaction of the technological energy characteristics of the formation of physical capital with the intergenerational transmission of natural resources. The general message of the paper is that the technological assumptions about the energy-intensity differences among sectors should be taken with caution. This is particularly important in dynamic models involving exhaustible energy resources.

In our model we have considered a number of simplifications for the sake of analytical tractability. We focused on a rather extreme case of sectoral energy-intensity differences. One could extend our set-up by including the energy input as well in the technology of consumption goods, thus allowing for the possibility of endogenous allocation of energy among sectors. This would result in more sophisticated cases of complex dynamics, adding to the problem the importance of the share of energy inputs in the production consumption goods. Notwithstanding, even if the model would be more realistic, from a general perspective the essence of the paper remains the same. Another simplification was to focused on exhaustible resources, which are known to impose serious limitations to economic growth. However, our framework can be also applied to study the case of renewables by setting $\Pi>0$ in the general model. This would create richer dynamics, where the convergence to steady-states without exhaustibility (see Proposition 2) may be possible since resources can naturally reproduce.

Other extensions are also worth pursuing. It would be interesting to further investigate the role played by the property rights. Considering the definition of resources assets in (1), it is easy to see that the initial (endogenous) extraction $x_{0}$ determines the initial allocation $a_{0}$ for an initial endowment of natural resources $E_{0}$. Therefore, Proposition 3 additionally shows that the initial allocation of resources property rights can be crucial to ensure that resources depletion does not occur in finite time (i.e., "tragedy of the commons" in the context of our model). From the perspective of the social welfare, one could widen our approach to study to what extend the (re)allocation of property rights would eliminate the intergenerational externalities and, thus, implement the social optimum. Finally, another line to explore is the characterization of specific "non-classical" evolution paths of extraction and energy prices. As observed before, Proposition 3 provides necessary conditions for this prospect. The analysis of the explicit (complex) solutions of the system presented in Proposition 1 would transform our conditions into necessary and sufficient. Additional economic mechanisms may arise behind those particular dynamics.

Acknowledgements We are thankful to Eric Strobl, Cuong Le Van and Ingmar Schumacher for their comments. The Associate Editor of this Journal and three anonymous referees provided suggestions that helped to make substantial improvements to the paper. Agustín Pérez-Barahona acknowledges financial support from the Chaire Développement Durable (Ecole Polytechnique - EDF) and the Labex MME-DII.

\section{Appendix A: Dynamical System}

In this appendix we present the details to derive the system (20)-(21) from the equations (1)-(17). Let us first describe the steps to obtain Eq. (18) in Sect. 3. Using the law of motion of the stock of energy resources (1), one can rewrite the budget constraint of the young as follows: 


$$
c_{t}+s_{t}+\frac{P_{t} e_{t+1}}{\Pi}=w_{t} .
$$

Similarly, considering the non-arbitrage condition (6) aboutx the prices of the asset and extracted energy, we can rewrite the budget constraint of the old as follows:

$$
e_{t+1}=\frac{d_{t+1}}{P_{t+1}}-\frac{R_{t+1} s_{t}}{P_{t+1}} .
$$

Taking (A.2) into the new formulation of the budget constraint of the young (A.1), we obtain:

$$
c_{t}+s_{t}+\frac{P_{t} d_{t+1}}{P_{t+1} \Pi}-\frac{R_{t+1} P_{t} s_{t}}{\Pi P_{t+1}}=w_{t} .
$$

Using the FOC (5) of the households' utility maximization, the above equation reduces to

$$
c_{t}+\frac{d_{t+1}}{R_{t+1}}=w_{t}
$$

Moreover, taking (4), this expression becomes

$$
c_{t}=\frac{w_{t}}{(1+\beta)} .
$$

From the budget constraint of the old (A.2), we can obtain the following equation for the savings:

$$
s_{t}=\frac{d_{t+1}}{R_{t+1}}-\frac{P_{t+1} e_{t+1}}{R_{t+1}} .
$$

In addition, considering the equality of savings and investment and provided that there is no population growth, Eq. (12) can be rewritten to obtain the savings:

$$
s_{t}=B_{t+1}^{\frac{-\theta}{1-\theta}} x_{t+1}^{\frac{-\theta}{1-\theta}} k_{t+1}^{\frac{1}{1-\theta}} .
$$

Equating (A.6) and (A.7) and considering the law of motion of the stock of energy resources (1), we establish the following equation:

$$
k_{t+1}^{\frac{1}{1-\theta}} B_{t+1}^{\frac{-\theta}{1-\theta}} x_{t+1}^{\frac{-\theta}{1-\theta}}=\frac{d_{t+1}}{R_{t+1}}-\frac{P_{t+1} \Pi\left(e_{t}-x_{t}\right)}{R_{t+1}} .
$$

Taking (A.4) and (A.5) above and using (5), (A.8) becomes:

$$
k_{t+1}^{\frac{1}{1-\theta}} B_{t+1}^{\frac{-\theta}{1-\theta}} x_{t+1}^{\frac{-\theta}{1-\theta}}=\frac{\beta w_{t}}{(1+\beta)}+P_{t}\left(x_{t}-e_{t}\right)
$$

From the FOC (10) of the firms' maximization problem in the final good sector, together with the energy price equation (16) and the condition (6), Eq. (A.9) can be reformulated as:

$$
k_{t+1}^{\frac{1}{1-\theta}} B_{t+1}^{\frac{-\theta}{1-\theta}} x_{t+1}^{\frac{-\theta}{1-\theta}}=\frac{\beta(1-\alpha) y_{t}}{(1+\beta)}+\theta \alpha y_{t}-\theta \alpha \frac{y_{t} e_{t}}{x_{t}} .
$$

Defining $\sigma \equiv \beta /(1+\beta)$ we obtain the equation (18) of the paper:

$$
k_{t+1}^{\frac{1}{1-\theta}}=B_{t+1}^{\frac{\theta}{1-\theta}} x_{t+1}^{\frac{\theta}{1-\theta}}\left[\sigma(1-\alpha)+\theta \alpha\left(1-\frac{e_{t}}{x_{t}}\right)\right] y_{t} .
$$

Let us specify the steps to compute Eq. (19). From (5) and (6) of the households' optimization problem and the energy price equation (16), we can set the interest rate:

$$
R_{t}=\Pi \frac{y_{t+1}}{y_{t}} \frac{x_{t}}{x_{t+1}}
$$


By inserting in this expression the FOC (9) of firms' maximization problem and the condition (17), we derive Eq. (19):

$$
\frac{\Pi k_{t+1}^{\frac{1}{1-\theta}} x_{t}}{y_{t} x_{t+1}}=\alpha(1-\theta) B_{t+1}^{\frac{\theta}{1-\theta}} x_{t+1}^{\frac{\theta}{1-\theta}} .
$$

Finally, we will show how substituting for $k_{t+1}$ in (18) and (19) yields the Eq. (20). Plugging the left hand side of (19) into (18), we get the following condition:

$$
\frac{x_{t+1}}{x_{t}} \frac{\alpha(1-\theta)}{\Pi}=\left[\sigma(1-\alpha)+\theta \alpha\left(1-\frac{e_{t}}{x_{t}}\right)\right] \text {. }
$$

Then, explicitly writing $\sigma \equiv \beta /(1+\beta)$, we obtain Eq. (20) in the model:

$$
x_{t+1}=\Pi\left(\frac{\frac{\beta}{(1+\beta)}(1-\alpha)+\alpha \theta}{\alpha(1-\theta)}\right) x_{t}-\frac{\Pi \theta}{(1-\theta)} e_{t} .
$$

Equation (20) governs the evolution the extraction flow while the law of motion of the natural resources is described by $e_{t+1}=\Pi\left(e_{t}-x_{t}\right)$ from condition (1).

\section{Appendix B: Proof of Proposition 2}

A steady-state $\left(x^{*}, e^{*}\right)$ of the economy is a fixed point of the system stated in Proposition 1 , where $x_{t+1}=x_{t}=x^{*}$ and $e_{t+1}=e_{t}=e^{*}$. We then obtain the Eqs. (22) and (23). Since $\Pi \geq 1, \psi_{1}$ and $\psi_{4}>0$, and $\psi_{2}$ and $\psi_{3}<0$, we can identify from the system of this proposition the following two sets of parameters, together with the characteristics of the corresponding steady-states:

(i) If $\left\{\psi_{1}=1\right\}$ or $\left\{\psi_{1} \neq 1\right.$ and $\left.\psi_{4}=1\right\}$ or $\left\{\psi_{1} \neq 1, \psi_{4} \neq 1\right.$ and $\left.\left(1-\psi_{4}\right) \neq \frac{\psi_{2} \psi_{3}}{\left(1-\psi_{1}\right)}\right\}$ there is a unique steady-state $\left(x^{*}, e^{*}\right)=(0,0)$.

(ii) If $\left\{\psi_{1} \neq 1, \psi_{4} \neq 1\right.$ and $\left.\left(1-\psi_{4}\right)=\frac{\psi_{2} \psi_{3}}{\left(1-\psi_{1}\right)}\right\}$ there is a continuum of steady-states such that $x^{*}=\frac{\psi_{2}}{1-\psi_{1}} e^{*}$.

Notice that sets (i) and (ii) comprise all possible parameter combinations.

\section{Appendix C: Proof of Proposition 3}

Let us first consider a set of intermediate outcomes. With these results in hand, we can then complete the proof of Proposition 3.

\section{Appendix C.1: Intermediate Results}

Claim 1 For non-renewable resources the discriminant $\Delta \equiv\left(1+\psi_{1}\right)^{2}-4\left(\psi_{1}+\psi_{2}\right)$ cannot be zero. In fact,

$$
\Delta>(<) 0 \Leftrightarrow 2(1-2 \theta)-\tilde{\beta}<(>) \frac{1}{\tilde{\beta}} .
$$

Moreover, for real eigenvalues, $\lambda_{1}>\lambda_{2}$.

Proof Let us prove that $\Delta \neq 0$. By contradiction, we assume that $\Delta=0$. Therefore $(1+$ $\left.\psi_{1}\right)^{2}=4\left(\psi_{1}+\psi_{2}\right)$. Since $\psi_{1}=\frac{\tilde{\beta}+\theta}{1-\theta}$ and $\psi_{2}=-\frac{\theta}{(1-\theta)}$, the previous condition is equivalent 
to $\left(\frac{1+\tilde{\beta}}{1-\theta}\right)^{2}=\frac{4 \tilde{\beta}}{1-\theta}$. This is possible iff $\tilde{\beta}=(1-2 \theta) \pm \sqrt{(1-2 \theta)^{2}-1}$. Since $(1-2 \theta)^{2}<1$, $\tilde{\beta}$ would be a complex number. This conclusion however contradicts our definition of $\tilde{\beta}$, which is a real number. We can indeed state that $\Delta>(<) 0$ iff $2(1-2 \theta)-\tilde{\beta}<(>) \frac{1}{\tilde{\beta}}$. Finally, from the expression of the real eigenvalues, we can directly observe that $\lambda_{1}>\lambda_{2}$ since $\psi_{1}>0$ and $\Delta \neq 0$

Claim 2 Whether the dynamics are complex or not depends on the following parameter combinations:

1. For $\frac{1}{2} \leq \theta<1$ or $\left\{0<\theta<\frac{1}{2}\right.$ and $\left.\tilde{\beta} \geqslant 2(1-2 \theta)\right\}$ the dynamics are non-complex.

2. For $\frac{1}{4} \leq \theta<\frac{1}{2}$ and $\tilde{\beta}<2(1-2 \theta)$ the dynamics are non-complex.

3. For $0<\theta<\frac{1}{4}$ and $\tilde{\beta}+\frac{1}{\tilde{\beta}}>2(1-2 \theta)$ the dynamics are non-complex.

4. For $0<\theta<\frac{1}{4}$ and $\tilde{\beta}+\frac{1}{\tilde{\beta}}<2(1-2 \theta)$ the dynamics are complex.

Proof The dynamics are (non-)complex iff $\Delta(>)<0$.

1. For $\frac{1}{2} \leq \theta<1$ or $\left\{0<\theta<\frac{1}{2}\right.$ and $\left.\tilde{\beta} \geqslant 2(1-2 \theta)\right\}$, it is easy to verify that, since $\tilde{\beta}>0$, $2(1-2 \theta)-\tilde{\beta}<\frac{1}{\tilde{\beta}}$. Therefore, from Claim $1, \Delta>0$.

2. By contradiction. Let us assume, for $\frac{1}{4} \leq \theta<\frac{1}{2}$ and $\tilde{\beta}<2(1-2 \theta)$, that $\Delta<0$. Claim 1 would then imply that $2(1-2 \theta)>\frac{1}{\tilde{\beta}}+\tilde{\beta}$. Therefore, since all terms are nonnegative, we conclude that $2(1-2 \theta)>\frac{1}{\tilde{\beta}}$ and $2(1-2 \theta)>\tilde{\beta}$. This can be rewritten as $2(1-2 \theta)>\tilde{\beta}>\frac{1}{2(1-2 \theta)}$. The condition holds iff $4(1-2 \theta)^{2}>1$. This reduces to $2(1-2 \theta)>1$ since $(1-2 \theta)>0$. Hence $\theta<\frac{1}{4}$, which contradicts our initial assumption on $\theta$.

3. It follows from Eq. (C.1).

4. It follows from Eq. (C.1).

Finally notice that the case $\tilde{\beta}+\frac{1}{\tilde{\beta}}=2(1-2 \theta)$ is not possible since $\Delta \neq 0$ (see Claim 1)

Claim 3 Considering the definition of $\Delta$ in Claim 1, we can establish the following results:

1. If $\frac{1}{2} \leq \theta<1$ then $1-\frac{\sqrt{\Delta}}{2}<0$.

2. For non-complex dynamics, $1+\frac{\sqrt{\Delta}}{2}>\frac{\left(1+\psi_{1}\right)}{2}$.

3. If $\frac{1}{2} \leq \theta<1$ then $\frac{\left(1+\psi_{1}\right)}{2}>1-\frac{\sqrt{\Delta}}{2}$.

4. If $0<\theta<\frac{1}{2}$ and $\tilde{\beta} \geqslant 2(1-2 \theta)$ then $\frac{\left(1+\psi_{1}\right)}{2}>1-\frac{\sqrt{\Delta}}{2}$.

5. If $\frac{1}{4} \leq \theta<\frac{1}{2}$ and $\tilde{\beta}<2(1-2 \theta)$ then $1-\frac{\sqrt{\Delta}}{2}>0$.

6. If $\frac{1}{4} \leq \theta<\frac{1}{2}$ and $\tilde{\beta}<2(1-2 \theta)$ then $\frac{\left(1+\psi_{1}\right)}{2}>1-\frac{\sqrt{\Delta}}{2}$.

\section{Proof}

1. Let us first show that $1-\frac{\sqrt{\Delta}}{2} \neq 0$. Suppose the contrary, i.e., $1=\frac{\sqrt{\Delta}}{2}$. Then $\tilde{\beta}=$ $(1-2 \theta) \pm 2 \sqrt{2 \theta^{2}-3 \theta+1}$. For $\theta=1 / 2$, the coefficient $\tilde{\beta}=0$ and so we find a contradiction because $\tilde{\beta}>0$. Let us study $1 / 2<\theta<1$. It can be verified that in this case $2 \theta^{2}-3 \theta+1 \neq 0: 2 \theta^{2}-3 \theta+1=0$ for $\theta=0$ or $\theta=1 / 2$. We can actually proof that $2 \theta^{2}-3 \theta+1<0$ : assuming that $2 \theta^{2}-3 \theta+1>0,3<\frac{1}{\theta}+2 \theta$; however, for $1 / 2<\theta<1$, it is easy to check that $3>\frac{1}{\theta}+2 \theta$. This result implies that $\tilde{\beta}$ is 
complex, which is a contradiction and, consequently, $1-\frac{\sqrt{\Delta}}{2} \neq 0$. We can show in fact that $1-\frac{\sqrt{\Delta}}{2}<0$. Let us assume the contrary, $1-\frac{\sqrt{\Delta}}{2}>0$. This would imply that $4(1-\theta)^{2}-1>\tilde{\beta}[\tilde{\beta}-2(1-2 \theta)]$. However, for $\frac{1}{2} \leq \theta<1$, the RHS $>0$ while LHS $<0$. We then get a contradiction.

2. By contradiction. Let us assume $1+\frac{\sqrt{\Delta}}{2} \leq \frac{\left(1+\psi_{1}\right)}{2}$. This is equivalent to $\left(\psi_{1}-1\right)^{2} \geq$ $\Delta(>0)$. Since $\Delta=\left(1+\psi_{1}\right)^{2}-4\left(\psi_{1}+\psi_{2}\right)$, the previous condition would imply that $0 \geq-4 \psi_{2}$. But this is impossible because $\psi_{2}<0$.

3. From statement 1 of this claim, we already know that $1-\frac{\sqrt{\Delta}}{2}<0$ if $\frac{1}{2} \leq \theta<1$. Our result is then verified since $\psi_{1}>0$.

4. By contradiction. Let us consider that $\frac{\left(1+\psi_{1}\right)}{2} \leq 1-\frac{\sqrt{\Delta}}{2}$. This would imply that $\psi_{1}-1 \leq$ $-\sqrt{\Delta}$. We know that $\psi_{1}-1=\frac{\tilde{\beta}-(1-2 \theta)}{1-\theta}$, which is strictly positive since $\tilde{\beta} \geq 2(1-2 \theta)>$ $(1-2 \theta)>0$. We then get a contradiction since $-\sqrt{\Delta}<0$.

5. Let us first show that $1-\frac{\sqrt{\Delta}}{2} \neq 0$. Suppose the contrary, i.e., $1=\frac{\sqrt{\Delta}}{2}$. Then $\tilde{\beta}=$ $(1-2 \theta) \pm 2 \sqrt{2 \theta^{2}-3 \theta+1}$. For $\frac{1}{4} \leq \theta<\frac{1}{2}$ one can verify that $2 \theta^{2}-3 \theta+1>0$ and $(1-2 \theta)>0$. We can show that $\tilde{\beta}=(1-2 \theta)+2 \sqrt{2 \theta^{2}-3 \theta+1}$ is impossible: let us rewrite $\tilde{\beta}=(1-2 \theta)+\sqrt{(1-2 \theta)^{2}+\left[4(1-\theta)^{2}-1\right]}$; it is easy to check that $0<\tilde{\beta}<2(1-2 \theta)<(1-2 \theta)+\sqrt{(1-2 \theta)^{2}+a}$ for $a>0$; recalling $a=4(1-\theta)^{2}-1$, since $4(1-\theta)^{2}-1>0$ for $0<\theta<1 / 2$, the previous expression provides the contradiction $\tilde{\beta}<\tilde{\beta}$. Similarly, $\tilde{\beta}=(1-2 \theta)-2 \sqrt{2 \theta^{2}-3 \theta+1}$ cannot hold either: since $\tilde{\beta}>0$, this would imply that $(1-2 \theta)>\sqrt{(1-2 \theta)^{2}+\left[4(1-\theta)^{2}-1\right]}$; however, $(1-2 \theta)<\sqrt{(1-2 \theta)^{2}+a}$ for $a>0$; as above, recalling $a=4(1-\theta)^{2}-1(>0)$ gives us a contradiction.

We know now that $1-\frac{\sqrt{\Delta}}{2} \neq 0$. In order to show that $1-\frac{\sqrt{\Delta}}{2}>0$, let us assume $1-\frac{\sqrt{\Delta}}{2}<0$. This would imply that $\tilde{\beta}[\tilde{\beta}-2(1-2 \theta)]>4(1-\theta)^{2}-1$. But this is not possible since the LHS $<0$ and the RHS $>0$.

6. By contradiction. We assume $\frac{\left(1+\psi_{1}\right)}{2} \leq 1-\frac{\sqrt{\Delta}}{2}$, which would imply $\left(\psi_{1}-1\right) \leq-\sqrt{\Delta}$. On the one hand, $-\sqrt{\Delta}<0$ because we are in a situation of non-complex dynamics. On the other hand, $\left(\psi_{1}-1\right)=\frac{\tilde{\beta}-(1-2 \theta)}{1-\theta}$, where we can identify two cases for $\tilde{\beta}<2(1-2 \theta)$ : (i) $(1-2 \theta) \leq \tilde{\beta}<2(1-2 \theta)$ and (ii) $\tilde{\beta}<(1-2 \theta)<2(1-2 \theta)$. Case (i) yields a contradiction since $\left(\psi_{1}-1\right)>0$. Case (ii) would imply $\left(\psi_{1}-1\right)^{2} \geq \Delta$, which is impossible for non-complex dynamics (see proof of Claim 3, statement 2).

\section{Appendix C.2: Proof of Proposition 3}

Proof For exhaustible resources $\Pi=1$. If $\left(1+\psi_{1}\right)^{2} \geq 4\left(\psi_{1}+\psi_{2}\right)$ the two eigenvalues of $\Psi$ are real and given by $\lambda_{1,2}=\frac{\left(1+\psi_{1}\right)}{2} \pm \frac{1}{2} \sqrt{\left(1+\psi_{1}\right)^{2}-4\left(\psi_{1}+\psi_{2}\right)}$. Moreover, $\lambda_{1,2}>0$ since the $\operatorname{det}(\Psi)>0$ and $\operatorname{Tr}(\Psi)>1 .{ }^{13}$ This implies that non-monotonic dynamics is only possible if the eigenvalues are complex: if $\left(1+\psi_{1}\right)^{2}<4\left(\psi_{1}+\psi_{2}\right), \lambda_{1,2}=\frac{\left(1+\psi_{1}\right)}{2} \pm$ $i \frac{1}{2} \sqrt{4\left(\psi_{1}+\psi_{2}\right)-\left(1+\psi_{1}\right)^{2}}$. Let us first study the case of non-complex dynamics. We distinguish four different sets of parametrization: $\frac{1}{2} \leq \theta<1$ (set 1); $0<\theta<\frac{1}{2}$ and

13 Notice that $\lambda_{1} \lambda_{2}=\operatorname{det}(\Psi)=\psi_{1}+\psi_{2}>0$. Since $\operatorname{det}(\Psi)>0, \lambda_{1,2} \neq 0$ and their sign coincide. Moreover, $\lambda_{1}+\lambda_{2}=\operatorname{Tr}(\Psi)=1+\psi_{1}>1$. We can then conclude that both eigenvalues are strictly positive. 
$\tilde{\beta} \geq 2(1-2 \theta)($ set 2$) ; \frac{1}{4} \leq \theta<\frac{1}{2}$ and $\tilde{\beta}<2(1-2 \theta)$ (set 3); and $0<\theta<\frac{1}{4}$ and $\tilde{\beta}<2(1-2 \theta)$ (set 4$)$. Let us study each of these sets.

Set 1: For $\frac{1}{2} \leq \theta<1$, the dynamics are non-complex as the discriminant $\Delta \equiv$ $\left(1+\psi_{1}\right)^{2}-4\left(\psi_{1}+\psi_{2}\right)>0$ (see Claim 2 in Appendix). Since $\lambda_{1,2}>0$, comparing $\lambda_{1}$ and $\lambda_{2}$ with 1 , one should observe that the dynamics are stable iff both eigenvalues are smaller than one. This is equivalent to $\frac{\left(1+\psi_{1}\right)}{2}<1-\frac{\sqrt{\Delta}}{2}$. However this is impossible because $\frac{\left(1+\psi_{1}\right)}{2}>1-\frac{\sqrt{\Delta}}{2}$ (see Claim 3 in Appendix). Therefore, stability cannot occur.

Let us consider the possibility of saddle equilibrium and (monotone) unstable dynamics. The steady-state is saddle-path stable iff the two eigenvalues are on the different side of one. As $\lambda_{1,2}>0$, this condition is equivalent to $\lambda_{1}>1$ and $\lambda_{2}<1$ (notice that, from Claim $1, \lambda_{1}>\lambda_{2}$ ). Taking the expression of $\lambda_{1,2}$, the condition reduces to $1-\frac{\sqrt{\Delta}}{2}<\frac{\left(1+\psi_{1}\right)}{2}<1+\frac{\sqrt{\Delta}}{2}$. In contrast to that, the equilibrium dynamics are unstable iff $\lambda_{1}>1$ and $\lambda_{2} \geq 1$. This is equivalent to check that $\frac{\left(1+\psi_{1}\right)}{2} \geq 1+\frac{\sqrt{\Delta}}{2}$. Since the dynamics are non-complex for $\frac{1}{2} \leq \theta<1$, we can conclude from Claim 3 that $\frac{\left(1+\psi_{1}\right)}{2}<1+\frac{\sqrt{\Delta}}{2}$. We have also proved in Claim 3 that $\frac{\left(1+\psi_{1}\right)}{2}>1-\frac{\sqrt{\Delta}}{2}$. Therefore, $1-\frac{\sqrt{\Delta}}{2}<\frac{\left(1+\psi_{1}\right)}{2}<1+\frac{\sqrt{\Delta}}{2}$ and, consequently, the steady-state is a saddle.

Set 2: For $0<\theta<\frac{1}{2}$ and $\tilde{\beta} \geq 2(1-2 \theta)$ the dynamics are non-complex (Claim 2). Therefore, from Claim 3, $\frac{\left(1+\psi_{1}\right)}{2}<1+\frac{\sqrt{\Delta}}{2}$. From the same claim we also know that, if $0<\theta<\frac{1}{2}$ and $\tilde{\beta} \geq 2(1-2 \theta), \frac{\left(1+\psi_{1}\right)}{2}>1-\frac{\sqrt{\Delta}}{2}$. We can then conclude that $1-\frac{\sqrt{\Delta}}{2}<\frac{\left(1+\psi_{1}\right)}{2}<1+\frac{\sqrt{\Delta}}{2}$, thus the steady-state is a saddle.

Set 3: We know from Claim 3 that, if $\frac{1}{4} \leq \theta<\frac{1}{2}$ and $\tilde{\beta}<2(1-2 \theta), 1-\frac{\sqrt{\Delta}}{2}<\frac{\left(1+\psi_{1}\right)}{2}$. Moreover $\frac{\left(1+\psi_{1}\right)}{2}<1+\frac{\sqrt{\Delta}}{2}$ because we are in a case on non-complex dynamics (see Claim 3). Therefore, as above, the steady-state is a saddle.

Set 4: It is easy to verify that the statements 5 and 6 in Claim 3 are also valid for $0<\theta<\frac{1}{4}$ and $\tilde{\beta}<2(1-2 \theta)$ if the dynamics are non-complex. Following Claim 2, we are in a case of non-complex dynamics. We can then conclude that $1-\frac{\sqrt{\Delta}}{2}<\frac{\left(1+\psi_{1}\right)}{2}<$ $1+\frac{\sqrt{\Delta}}{2}$, thus the steady-state is a saddle.

Let us finish the proof with the case of complex dynamics. From Claim 2 we know that (i) in the proposition corresponds to complex dynamics. Taking the formulas for $\lambda_{1,2}$ above, one can verify that $\left|\lambda_{1}\right|=\left|\lambda_{2}\right|=\sqrt{\frac{\tilde{\beta}}{(1-\theta)}}$. Comparing $\left|\lambda_{1}\right|=\left|\lambda_{2}\right|$ with 1 , we can directly conclude that the dynamics are stable iff the modulus of both eigenvalues is smaller than one. This condition is in fact equivalent to $\tilde{\beta}<(1-\theta)$. Similarly, the dynamics are unstable iff the modulus of both eigenvalues is larger than one, which corresponds to $\tilde{\beta}>(1-\theta)$.

\section{References}

Acemoglu D (2009) Introduction to modern economic growth. Princeton University Press, Princeton Agnani B, Gutierrez M, Iza A (2005) Growth in overlapping generation economies with non-renewable resources. J Environ Econ Manag 50:387-407

Ahrens W, Sharma V (1997) Trends in natural resource commodity prices: deterministic or stochastic? J Environ Econ Manag 33:59-74 
Altonji J, Hayashi F, Kotlikoff L (1992) Is the extended family altruistically linked? Direct tests using micro data. Am Econ Rev 82:1177-1198

Balestra C (2007) Environmental externalities and fiscal policy in an overlapping-generation model. Mimeo, New York

Barnett H, Morse C (1963) Scarcity and growth: the economics of natural resource availability. Johns Hopkins University Press for Resources for the Future, Baltimore

Bednar-Friedl B, Farmer K (2013) Time consuming resource extraction in an OLG economy with capital. J Econ 110:203-224

Brookes L (2000) Energy efficiency fallacies revisited. Energy Policy 28:355-366

Bullard C III, Herendeen R (1975) The energy cost of goods and services. Energy Policy 3(4):268-278

Costanza R (1980) Embodied energy and economic valuation. Science 210(12):1219-1224

Dasgupta P, Heal G (1974) The optimal depletion of exhaustible resources. Rev Econ Stud 41:3-28

Dasgupta P, Heal G (1979) Economic theory and exhaustible resources. Cambridge University Press, Cambridge

de la Croix D, Michel P (2004) A theory of economic growth: dynamics and policy in overlapping generations. Cambridge University Press, Cambridge

Ecorys (2009) Study on European energy-intensive industries - the usefulness of estimating sectoral price elasticities. Ecorys SCS Group for the Directorate-General Enterprise and Industry, European Commission, Rotterdam

Farmer K (2000) Intergenerational natural-capital equality in an overlapping-generations model with logistic regeneration. J Econ 72:129-152

Ferraro D, Peretto P (2014) Commodity prices, long-run growth and fiscal vulnerability. Mimeo, New York

Greening L, Greene D, Difiglio C (2000) Energy efficiency and consumption—the rebound effect—a survey. Energy Policy 28:389-401

Heal G (1976) The relationship between price and extraction cost for resource with a backstop technology. Bell J Econ 7:317-378

Hotelling H (1931) The economics of exhaustible resources. J Polit Econ 39:137-75

Kijima M, Nishide K, Ohyama A (2010) Economic models for the environmental Kuznets curve: a survey. J Econ Dyn Control 34:1187-1201

Kim I, Lounging P (1992) The role of energy in real business cycle models. J Monet Econ 29:173-189

Koskela E, Ollikainen M, Puhakka M (2002) Renewable resources in an overlapping generations economy without capital. J Environ Econ Manag 43(3):497-517

Krautkramer J (1998) Nonrenewable resource scarcity. J Econ Lit 36:2065-2107

Le T, Le Van C (2016) Transitional dynamics in an R\&D-based growth model with natural resources. Math Soc Sci 82:1-17

Le T, Le Van C (2017) Renewable or non-renewable resources: which generate more growth from R\&D? Econ Model (forthcoming)

Lutz K (2008) The economic effects of energy price shocks. J Econ Lit 46(4):871-909

Miketa A (2001) Analysis of energy intensity developments in manufacturing sectors in industrialized and developing countries. Energy Policy 29:769-775

Mourmouras A (1991) Competitive equilibria and sustainable growth in a life-cycle model with natural resources. Scand J Econ 93(4):585-591

Olson L, Knapp K (1997) Exhaustible resource allocation in an overlapping generations economy. J Environ Econ Manag 32:277-292

Padilla E (2002) Intergenerational equity and sustainability. Ecol Econ 41:69-83

Pérez-Baharona A (2011) Nonrenewable energy resources as input for physical capital accumulation: a new approach. Macroecon Dyn 15(1):1-30

Pérez-Barahona A, Zou B (2006) A comparative study of energy saving technical progress in a vintage capital model. Resour Energy Econ 28(2):181-191

Pindyck R (1980) Uncertainty and exhaustible resource markets. J Polit Econ 88:1203-1225

Sen P (2016) Unilateral emission cuts and carbon leakages in a dynamic north-south trade model. Environ Resour Econ 64(1):131-152

Slade M (1982) Trends in natural-resource commodity prices: an analysis of the time domain. J Environ Econ Manag 9:122-137

Solow R (1974) Intergenerational equity and exhaustible resources. Rev Econ Stud 41:29-45

Stiglitz J (1974) Growth with exhaustible natural resources: efficient and optimal growth paths. Rev Econ Stud 41:123-137

Tahvonen O, Salo S (2001) Economic growth and transitions between renewable and nonrenewable energy resources. Eur Econ Rev 45:1379-1398 
United Nations Industrial Development Organization, UNIDO (2011) Industrial energy efficiency for sustainable wealth creation. United Nations, Vienna

Upadhyaya S (2010) Compilation of energy statistics for economic analysis, development policy and strategic research branch working papers. United Nations Industrial Development Organization, 01/2010

Valente S (2008) Sustainable development, renewable resources and technological progress. Environ Resour Econ 30:115-125 